\title{
Efficient Phase Contrast Microscopy Restoration Applied for Muscle Myotube Detection
}

\author{
Seungil Huh ${ }^{1,2}$, Hang $\mathrm{Su}^{2,3}$, Mei Chen ${ }^{4}$, and Takeo Kanade ${ }^{1,2}$ \\ 1 Lane Center for Computational Biology \\ 2 Robotics Institute, Carnegie Mellon University \\ 3 Department of Electronic Engineering, Shanghai Jiaotong University \\ 4 Intel Science and Technology Center on Embedded Computing
}

\begin{abstract}
This paper proposes a new image restoration method for phase contrast microscopy as a mean to enhance the quality of images prior to image analysis. Compared to state-of-the-art image restoration algorithms, our method has a more solid theoretical foundation and is orders of magnitude more efficient in computation. We validated the proposed method by applying it to automated muscle myotube detection, a challenging problem that has not been tackled without staining images. Results on 300 phase contrast microscopy images from three different culture conditions demonstrate that the proposed restoration scheme improves myotube detection, and that our approach is far more computationally efficient than previous methods.
\end{abstract}

\section{Introduction}

Vision-based analysis adopting phase contrast microscopy often has advantages over the use of fluorescent microscopy for the task of cell behavior and fate analysis; it enables continuous monitoring of intact cells in culture and it reduces the expenditure of consumable reagents as well as human labor. However, the analysis of phase contrast microscopy is not trivial because of the properties of the images: cells often lack distinctive textures, and artifacts, such as bright halos, often appear when cells form a cluster or undergo a certain process.

To address these issues, several image restoration methods have been proposed based on the image formation process of a phase contrast microscope [123]. These methods were devised to restore phase retardations at each point of a given image, based on which image analysis, such as cell region detection, can be more effectively performed. However, these restorations are often inaccurate due to the strong assumptions [12] or unable to generate restored images but can only produce features used for image analysis [3]. Furthermore, the high computational cost limits their applicability.

In this paper, we propose a new phase contrast microscopy image restoration algorithm that has a more solid theoretical foundation and is far more efficient than previous methods. More specifically, our method does not introduce any assumption in the modeling step and minimizes the computation complexity by employing the Wiener deconvolution algorithm [4]. As a result, our method 
produces more accurate restored images - not just features - and significantly outperforms previous methods in terms of computational efficiency.

We validated the restoration method by applying it to muscle myotube detection. Detection of muscle myotubes is important in two folds. First, it helps better understanding on the mechanism of muscle differentiation, which is required to improve the treatment of various muscular disorders associated with muscle loss, such as spinal muscular atrophy and muscular dystrophy [5]. In addition, precise detection of muscle myotubes that result from cell differentiation can automate the process of finding the optimal condition to keep stem cells from differentiating and thus losing self-renewal capability. To the best of our knowledge, there has not been published work on vision-based muscle myotube detection in phase contrast microscopy images.

\section{Previous Work}

Phase contrast microscopy is a popular optical microscopy technique that enhances the phase shift in light passing through a specimen and converts it into brightness change in images. According to [1], phase-contrast imaging can be modeled by two waves: the unaltered surround wave $l_{S}(x)$ and the diffracted wave $l_{D}(x)$, computed as

$$
\begin{aligned}
& l_{S}(x)=i \zeta_{p} A e^{i \beta} \\
& l_{D}(x)=\zeta_{c} A e^{i(\beta+\theta(x))} \delta(R)+\left(i \zeta_{p}-1\right) \zeta_{c} A e^{i\left(\beta+\theta\left(R_{x}\right)\right)} * \operatorname{Airy}(R)
\end{aligned}
$$

where $i^{2}=-1 ; A$ and $\beta$ are the illuminating wave's amplitude and phase before hitting the specimen plate, respectively; $\zeta_{p}$ and $\zeta_{c}$ are the amplitude attenuation factors by the phase ring and the specimen, respectively; $\theta(x)$ and $\theta\left(R_{x}\right)$ are the phase shifts caused by the specimen at location $x$ and its neighboring region $R_{x}$ with size $R$, respectively; $\delta(\cdot)$ is a $2 \mathrm{D}$ Dirac delta function; and $\operatorname{Airy}(R)$ is an obscured Airy pattern [6] with size $R$. A phase-contrast microscopy image $g$ can then be analytically modeled as a function of $\theta$ :

$$
\begin{aligned}
g(x) & =\left|l_{S}(x)+l_{D}(x)\right|^{2} \\
& =\left|\left(i \zeta_{p} A e^{i \beta}+\zeta_{c} A e^{i(\beta+\theta(x))}\right) \delta(R)+\left(i \zeta_{p}-1\right) \zeta_{c} A e^{i\left(\beta+\theta\left(R_{x}\right)\right)} * \operatorname{Airy}(R)\right|^{2} .
\end{aligned}
$$

To restore $\theta$ from $g$, so as to use $\theta$ instead of $g$ for image analysis, [1] applied the approximation $e^{i \theta(x)} \approx 1+i \theta(x)$ on the assumption that $\theta(x)$ is close to zero. Since this assumption is not valid for thick (and thus bright) cells, cells undergoing mitosis or apoptosis are often missed in the restored images [2]. Later, 2] generalized this model by assuming that $\theta(x)$ is close to a constant, which is not necessarily zero. This method is useful for the detection of particular cell regions, e.g., bright cell regions or dark cell regions; however, it cannot precisely restore phase retardations because the assumption that all phase retardations are close to one value is still generally not true. 
Recently, 3. applied the approximation

$$
e^{i \theta(x)} \approx \sum_{k=1}^{K} \psi_{k}(x) e^{i \theta_{k}}
$$

and showed that a phase contrast microscopy image $g$ can be modeled as a linear combination of $K$ diffraction patterns after flat-field correction, as follows:

$$
g=\sum_{k=1}^{K} \Psi_{k} *\left(\sin \theta_{k} \delta(R)+\left(\zeta_{p} \cos \theta_{m_{k}}-\sin \theta_{k}\right) * \operatorname{Airy}(R)\right)
$$

where $\theta_{k}$ is the $k$-th representative phase retardation, which is one of the $M$ equally distributed phases within $2 \pi$, i.e., $\left\{0, \frac{2 \pi}{M}, \cdots, \frac{2(M-1) \pi}{M}\right\}$. Given a phase contrast image $g$, by solving Eq. (5),$K$ coefficient matrices $\left(\Psi_{1}, \cdots, \Psi_{K}\right)$ are obtained. Although these coefficients can be used for image analysis, actual phase retardations may not be accurately estimated because solving for $\theta$ in Eq. (4) with obtained coefficients does not yield a right answer unless $\left|\sum_{k=1}^{K} \psi_{k}(x) e^{i \theta_{k}}\right|=$ 1, which is not guaranteed. In addition, Eq. (5) is an overdetermined system with $K$ times as many unknowns as equations. These unnecessarily many unknowns make the optimization problem difficult, but with little benefit.

\section{Restoration of Phase Contrast Microscopy}

In this section, we propose an effective and efficient restoration method for phase contrast microscopy and discuss the advantages of our method over the previous methods.

\subsection{Modeling of Phase Contrast Microscopy Imaging}

Eq. (3), the original phase-contrast model, can be expanded as follows:

$$
\begin{aligned}
g(x)= & \left|l_{S}+l_{D}\right|^{2}=\left(l_{S}+l_{D}\right) \cdot\left(l_{S}+l_{D}\right)^{*} \\
= & A^{2}\left[\left(\zeta_{p}{ }^{2}+\zeta_{c}{ }^{2}\right) \delta(R)-2 \zeta_{c}^{2} \operatorname{Airy}(R)+\left(\zeta_{p}^{2}+1\right) \zeta_{c}^{2} \operatorname{Airy}(R) * \operatorname{Airy}(R)+\right. \\
& \left.i \zeta_{p} \zeta_{c}\left(e^{-i \theta(x)}-e^{i \theta(x)}\right) \delta(R)+\zeta_{p} \zeta_{c}\left(\left(\zeta_{p}-i\right) e^{-i \theta\left(R_{x}\right)}+\left(\zeta_{p}+i\right) e^{i \theta\left(R_{x}\right)}\right) * \operatorname{Airy}(R)\right] \\
= & C_{1}+C_{2}\left[i\left(e^{-i \theta(x)}-e^{i \theta(x)}\right) \delta(R)+\left(\left(\zeta_{p}-i\right) e^{-i \theta\left(R_{x}\right)}+\left(\zeta_{p}+i\right) e^{i \theta\left(R_{x}\right)}\right) * \operatorname{Airy}(R)\right]
\end{aligned}
$$

where $C_{1}$ and $C_{2}$ are values that are not relevant to $\theta(x)$. Since $e^{i \theta(x)}=\cos (x)+$ $i \sin (x), e^{i \theta(x)}$ can be replaced with $\alpha(x)+i \beta(x)$ on the condition $\alpha(x)^{2}+\beta(x)^{2}=$ 1 , and then $g$ is reexpressed as:

$$
\begin{array}{r}
g(x)=C_{1}+C_{2}\left(2 \beta(x) \delta(R)+2\left(\zeta_{p} \alpha\left(R_{x}\right)-\beta\left(R_{x}\right)\right) * \operatorname{Airy}(R)\right) \\
\text { s.t. } \alpha(x)^{2}+\beta(x)^{2}=1 .
\end{array}
$$


After flat-field correction, we obtain the following model for the entire image.

$$
g=C\left(\zeta_{p} \alpha * \operatorname{Airy}(R)+\beta *(\delta(R)-\operatorname{Airy}(R))\right) \quad \text { s.t. } \alpha \otimes \alpha+\beta \otimes \beta=\mathbb{1}
$$

where $\alpha$ and $\beta$ are matrices with the same size of $g$ whose values at position $x$ are $\alpha(x)$ and $\beta(x)$, respectively, $\otimes$ is the element-wise matrix multiplication operator, $\mathbb{1}$ is the matrix with all 1 , and $C$ is a constant.

\subsection{Optimization Process for the Restoration}

Given $g$, in order to infer $\alpha$ and $\beta$ from this equation, we minimize the following objective:

$$
\begin{array}{r}
\min _{\alpha, \beta, C}\|g-C f\|_{F}^{2}, \quad f=\zeta_{p} \alpha * \operatorname{Airy}(R)+\beta *(\delta(R)-\operatorname{Airy}(R)) \\
\text { s.t. } \alpha \otimes \alpha+\beta \otimes \beta=\mathbb{1}
\end{array}
$$

where $\|\cdot\|_{F}$ denotes the Frobenius norm. Note that we also infer $C$ rather than manually setting it because all the parameters that required to determine $C$ are often not given. Once $\alpha$ and $\beta$ are obtained, phase retardation $\theta$ can be computed based on $e^{i \theta(x)}=\alpha(x)+i \beta(x)$.

In order to solve this optimization problem, we propose an iterative scheme using the Wiener deconvolution algorithm [4], which is an efficient way to perform deconvolution and has been popularly used for image deconvolution applications. The optimization procedure is as follows:

- Step 1. Initialize $\alpha$ and $\beta$ at random to satisfy $\alpha \otimes \alpha+\beta \otimes \beta=\mathbb{1}$.

- Step 2. Update $\alpha$ to minimize $\|g-C f\|_{F}^{2}$ with fixed $\beta$ by solving the following deconvolution problem via Wiener deconvolution.

$$
g-\beta *(C(\delta(R)-\operatorname{Airy}(R)))=\alpha *\left(C \zeta_{p} \operatorname{Airy}(R)\right) .
$$

- Step 3. Project $\alpha$ and $\beta$ onto the constraint space by multiplying $1 / \sqrt{\alpha^{2}+\beta^{2}}$.

- Step 4. Recalculate $C$ to minimize $\|g-C f\|_{F}^{2}$.

$$
C \leftarrow \frac{\operatorname{vec}(f) \cdot \operatorname{vec}(g)}{\operatorname{vec}(f) \cdot \operatorname{vec}(f)} .
$$

where $\operatorname{vec}(\cdot)$ is the vectorization operator.

- Step 5. Update $\beta$ to minimize $\|g-C f\|_{F}^{2}$ with fixed $\alpha$ by solving the following deconvolution problem via Wiener deconvolution.

$$
g-\alpha *\left(C \zeta_{p} \operatorname{Airy}(R)\right)=\beta *(C(\delta(R)-\operatorname{Airy}(R))) .
$$

- Step 6. Project $\alpha$ and $\beta$ in the same way as Step 3.

- Step 7. Recalculate $C$ in the same way as Step 4.

- Step 8. Repeat Steps 2 through 7 until $\|g-C f\|_{F}^{2}$ is not reduced any more.

Wiener deconvolution requires a parameter that indicates the signal-to-noise ratio of the original data 4]. In our experiments, we tested several values, namely $\{0,0.01, \cdots, 0.05\}$, and determined the parameter as the one that minimizes the final objective function value simply on the first image. 


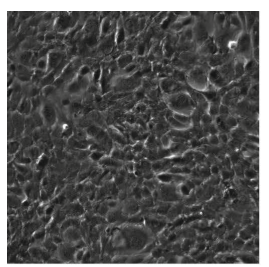

(a)

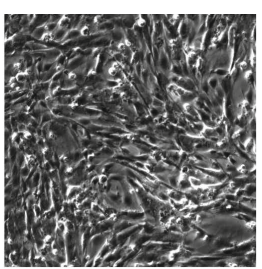

(b)

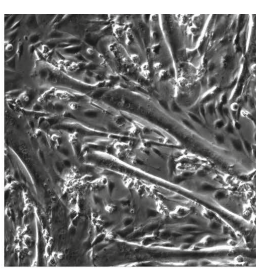

(c)

Fig. 1. The process of myotube formation: (a) single-nucleated myoblasts; (b,c) nascent and mature myotubes formed by the fusion of myoblasts, respectively

\subsection{Discussion on the Proposed Restoration Method}

The advantages of our method over the previous methods are in three folds:

First, our method can precisely restore phase retardations caused by cells without introducing any unreasonable approximation. On the other hand, previous methods either assume that phase retardations are close to a certain value [12] or adopt a linear approximation without appropriate constraints [3].

Second, our method is far more efficient than the previous methods in terms of computation time (time complexity of Wiener deconvolution vs. that of iterative deconvolution; in practice, a few seconds vs. several minutes for processing one image in a typical setting) and memory use (a few MB vs. a few GB). This is an important quality towards enabling real-time processing of time-lapse images.

Third, our method is theoretically more sound than the previous methods, particularly [3]. Since the model involves a lot more unknowns than equations, it might not be theoretically sound to infer the model via an iterative greedy scheme that alternates basis selection and coefficient calculation. In fact, the greedy scheme often selects different sets of bases in a different order (with repetition) for different images, when they do not show similar levels of cell density and maturity, so that feature sets from different images may not be consistent. On the other hand, our optimization is conducted in a standard manner.

One drawback of our method lies in fact that Wiener deconvolution cannot explicitly handle spatial or temporal smoothness terms incorporated into the objective function, unlike iterative deconvolution methods. This issue might be implicitly dealt with by applying smoothing during iterations.

\section{Muscle Myotube Detection}

This section introduces muscle myotube detection task as a testbed of our restoration method. During the differentiation of muscle stem cells, muscle myotubes are formed by the fusion of mononucleated progenitor cells known as myoblasts (See Fig 1). Given a phase contrast image containing both myoblasts and myotubes, the goal of muscle myotube detection is to identify the area where myotubes are located. This information is useful for measuring how far differentiation has proceeded and provides guidance for human intervention. 


\subsection{Myotube Detection Algorithm}

We examined two methods: pixel- and superpixel-based methods.

For the pixel-based method, after image restoration, we extract visual feature around each pixel in the restored image. We use rotation invariant local binary pattern $\left(\mathrm{LBP}^{\text {riu2 }}\right)$ [7, which is one of the most popular and effective texture features at present. For each pixel, we compute the distribution of different $\mathrm{LBP}^{r i u 2}$ in its neighboring region and use it as visual features of the pixel.

For the superpixel-based method, after image restoration, we perform superpixel segmentation using the entropy rate superpixel segmentation method [8]. Then for each superpixel, we compute the visual feature vector by computing the distribution of different $\mathrm{LBP}^{\text {riu2 }}$ within the superpixel.

After feature computation, we train a linear support vector machine over pixels or superpixels. For the superpixel-based method, given ground truth, the superpixels that contain more positive pixels than negative ones are used as positive samples and the rest as negative samples in the training phase. Testing is also conducted over superpixels; i.e., the pixels belonging to the same superpixel are determined to have the same label.

\section{Experiment}

\subsection{Data and Comparison}

Three sets of phase contrast images of mouse $\mathrm{C} 2 \mathrm{C} 12$ myoblasts were acquired under culture conditions with different amount $(100,500$, and $1000 \mathrm{ng} / \mathrm{mL})$ of IGF2, which accelerates differentiation. Each set contains 100 images; i.e., 300 images were obtained in total. Each image contains $640 \times 640$ pixels.

For comparison, immunofluorescence staining images capturing myotubes were acquired. Each staining image was reduced to a binary image by intensity thresholding. Note that binarized images include most of information on cell differentiation that biologists currently want to obtain via high-throughput screening. It is also worth mentioning that staining images are not the ground truth in that nuclei of myotubes are often not stained and the myotube boundary is not precise. Although imperfect, using staining images is a standard way for comparison since manual annotation is too time-consuming and often subjective.

The data and staining images will be available on the first author's home page (www.cs.cmu.edu/ seungilh).

\subsection{Experiments and Results}

We compare results of our myotube detection methods with those of the methods that do not adopt the restoration process. In these baseline methods, feature extraction was performed on phase contrast images, not the restored images. We perform 10-fold evaluation; for each set of images, we used 1 fold of images (10 images) for testing in turn and the rest for training. For each image, we compare the detection result with the staining image to compute precision and recall. 
Table 1. Myotube detection results in terms of F-measure. Our restoration method enhances myotube detection accuracy.

\begin{tabular}{|c|c|c|c|}
\hline & IGF2-100 & IGF2-500 & IGF2-1000 \\
\hline Phase contrast image+pixel & $0.32 \pm 0.06$ & $0.53 \pm 0.11$ & $0.67 \pm 0.10$ \\
\hline Restored image+pixel & $0.44 \pm 0.08$ & $0.68 \pm 0.09$ & $0.79 \pm 0.07$ \\
\hline Phase contrast image+superpixel & $0.61 \pm 0.07$ & $0.73 \pm 0.08$ & $0.80 \pm 0.08$ \\
\hline Restored image+superpixel & $\mathbf{0 . 6 5} \pm \mathbf{0 . 0 6}$ & $\mathbf{0 . 7 6} \pm \mathbf{0 . 0 4}$ & $\mathbf{0 . 8 6} \pm \mathbf{0 . 0 5}$ \\
\hline
\end{tabular}

Table 2. Performance comparison between our restoration method and the previous restoration method [3]. Computational time is computed for restoration methods.

\begin{tabular}{|l|c|c|c||c|}
\hline & IGF2-100 & IGF2-500 & IGF2-1000 & Time \\
\hline Our restoration+superpixel & $0.65 \pm 0.06$ & $0.76 \pm 0.04$ & $0.86 \pm 0.05$ & $2 \mathrm{sec}$ \\
\hline Restoration $[\underline{3}+$ superpixel & $0.63 \pm 0.08$ & $0.76 \pm 0.07$ & $0.86 \pm 0.06$ & $262 \mathrm{sec}$ \\
\hline
\end{tabular}
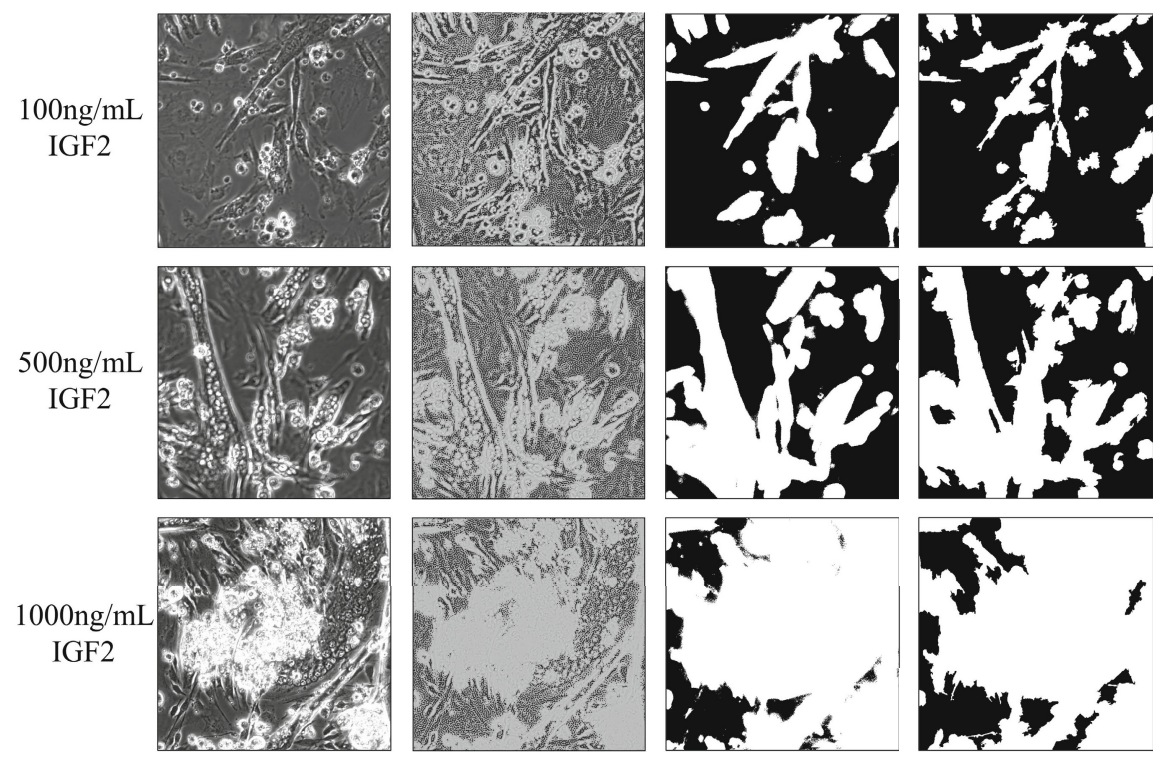

Fig. 2. Phase contrast images (1st column), restored images (2nd column), thresholded staining image (3rd column), and myotube detection results with the restoration and superpixel segmentation (4th column)

Over the entire 100 images for each set, we compute the average F-measure, which is the harmonic mean of precision and recall.

As shown in Table 1. when compared with the staining image, the method adopting both the restoration and superpixel segmentation achieves $65 \%$ to $86 \%$ accuracy in terms of F-measure 1 Myotube detection is more accurate under the

\footnotetext{
${ }^{1}$ It is reasonable to expect increased performance when the ground truth is used rather than staining images, which are imperfect so can possibly confuse the training model.
} 
condition with more amount of IGF2 because additional IGF2 leads to more mature myotubes the texture of which is more distinct from that of myoblasts. Applying the restoration scheme results in $12 \%$ to $15 \%$ gain in accuracy for the pixel-based method and $3 \%$ to $6 \%$ gain in accuracy for the superpixel-based method compared to the baseline methods. Fig. 2 shows examples of restoration images and myotube detection results.

Table 2 demonstrates that the proposed restoration method is considerably more efficient than the state-of-the-art restoration method [3]. Although the performances for myotube detection are comparable on these data sets, our method has advantages over the previous method including computational efficiency, capability to obtain restored images, and a more solid theoretical foundation.

\section{Conclusions}

In this paper, we propose a new image restoration method for phase contrast microscopy, which is theoretically more sound and computationally more efficient than previous methods. We also present a method for myotube detection that adopts the proposed restoration scheme and empirically validate the effectiveness and efficiency of the proposed restoration and myotube detection methods.

The next goal will be to monitor myotube formation over time. Though in this work, we focused on individual images, temporal information can be incorporated in several ways and it will result in a better performance for continuous monitoring of cell fate. We leave the empirical validation as future work.

\section{References}

1. Yin, Z., Kanade, T., Chen, M.: Understanding the phase contrast optics to restore artifact-free microscopy images for segmentation. Med. Image Anal. 16(5), 1047-1062 (2012)

2. Huh, S., Ker, D.F.E., Su, H., Kanade, T.: Apoptosis Detection for Adherent Cell Populations in Time-Lapse Phase-Contrast Microscopy Images. In: Ayache, N., Delingette, H., Golland, P., Mori, K. (eds.) MICCAI 2012, Part I. LNCS, vol. 7510, pp. 331-339. Springer, Heidelberg (2012)

3. Su, H., Yin, Z., Kanade, T., Huh, S.: Phase contrast image restoration via dictionary representation of diffraction patterns. In: Ayache, N., Delingette, H., Golland, P., Mori, K. (eds.) MICCAI 2012, Part III. LNCS, vol. 7512, pp. 615-622. Springer, Heidelberg (2012)

4. Gonzalez, R.C., Woods, R.E.: Digital Image Prcessing. Addison-Wesley Publishing Company, Inc. (1992)

5. Tedesco, F.S., Dellavalle, A., Diaz-Manera, J., Messina, G., Cossu, G.: Repairing skeletal muscle: regenerative potential of skeletal muscle stem cells. J. Clin. Invest. 120(1), 11-19 (2010)

6. Born, M., Wolf, E.: Principles of Optics, 6th edn. Pergamon Press (1980)

7. Ojala, T., Pietikäinen, M., Mäenpää, T.T.: Multiresolution gray-scale and rotation invariant texture classification with local binary pattern. IEEE Trans. Pattern. Anal. Mach. Intell. 24(7), 971-987 (2002)

8. Liu, M.-Y., Tuzel, O., Ramalingam, S., Chellappa, R.: Entropy Rate Superpixel Segmentation. In: Proc. CVPR, pp. 2097-2104 (2011) 